PHYSICAL REVIEW E 70, 061403 (2004)

\title{
Interaction of proteins with spherical polyelectrolyte brushes in solution as studied by small-angle x-ray scattering
}

\author{
S. Rosenfeldt, A. Wittemann, and M. Ballauff* \\ Physikalische Chemie I, University of Bayreuth, 95440 Bayreuth, Germany \\ E. Breininger, J. Bolze, and N. Dingenouts ${ }^{\dagger}$ \\ Polymer-Institut, University of Karlsruhe, Kaiserstrasse 12, 76128 Karlsruhe, Germany
}

(Received 9 June 2004; published 10 December 2004)

\begin{abstract}
We use small-angle x-ray scattering (SAXS) as a tool to study the binding of proteins to spherical polyelectrolyte brushes (SPB) in situ. The SPB consists of a solid core of $\sim 100 \mathrm{~nm}$ diam onto which long polyelectrolyte chains [poly(styrene sulfonic acid, PSS) and poly(acrylic acid, PAA)] have been densely grafted. The proteins used in this investigation, Bovine Serum Albumine (BSA) and Bovine Pancreatic Ribonuclease A (RNase A), adsorb strongly to these SPB if the ionic strength is low despite their negative charge. Virtually no adsorption takes place at high ionic strength. SAXS demonstrates that both proteins are distributed within the brush. The findings reported here give further evidence that the strong adsorption of proteins to SPB is due to the "counterions release forces": The patches of positive charge on the surface of the proteins become multivalent counterions of the polyelectrolyte chains. Thus, a concomitant number of co- and counterions is thereby released and the entropy of the entire system is increased. The repulsive Coulombic interaction as well as the steric repulsion between the proteins and the brush layer are counterbalanced by this effect. The data discussed here demonstrate that the adsorption of proteins in SPB presents a new principle for the immobilization of proteins.
\end{abstract}

DOI: 10.1103/PhysRevE.70.061403

PACS number(s): 82.70.-y, 68.43.-h, 87.15.-v, 87.68.+z

\section{INTRODUCTION}

The interaction of proteins with well-defined surfaces presents a very active field of research by now [1-3]. In many cases, adsorption of biomolecules onto solid surfaces needs to be avoided to prevent denaturation and loss of biological function [4]. On the other hand, nanoparticles carrying proteins may be of considerable technological interest [5]. The large surface offered by colloidal suspensions allows in principle the immobilization of a high amount of proteins, antibodies, or enzymes for a variety of possible applications. Clearly, a detailed understanding of the various factors governing the interaction of proteins with colloidal particles is the prerequisite of possible applications. In particular, adsorption onto solid surfaces may often lead to irreversible denaturation $[6,7]$ and strong deformation of the shape of the proteins [8]. Evidently, a strong interaction of the protein with the nanoparticle that is followed by its deformation must lead to a loss of most of the enzymatic activity $[6,9]$.

Recently, we have shown that spherical polyelectrolyte brushes (SPB) present a new class of carrier particles for proteins and enzymes [10-14]. These SPB consist of a core of solid poly(styrene) onto which long polyelectrolyte chains are grafted (see Fig. 1). The diameter of the core particles is of the order of $100 \mathrm{~nm}$. The chains are densely grafted so that the mean distance of the chains directly at the surface is much smaller than their contour length. In this way, the brush limit, i.e., the limit of strongly interacting chains affixed to a

\footnotetext{
*Electronic address: matthias.ballauff@uni-bayreuth.de

${ }^{\dagger}$ Electronic address: nico.dingenouts@polymer.uni-karlsruhe.de
}

solid surface, is attained $[15,16]$. Two types of polyelectrolyte chains have been used in this study: the weak polyelectrolyte poly(acrylic acid) (PAA) and the strong polyelectrolyte poly(styrene sulfonic acid) (PSS). Affixing weak polyelectrolyte chains leads to an annealed SPB because the number of charges depends on the $p \mathrm{H}$ present in the system [17]. Particles carrying strong polyelectrolytes are named quenched SPB because the charges do not depend on the $p \mathrm{H}$ in the solution [17]. The contour length of the polyelectrolyte chains that have been affixed by a "grafting-from technique" is of the order of $100 \mathrm{~nm}[18,19]$. Proteins such as, e.g.,

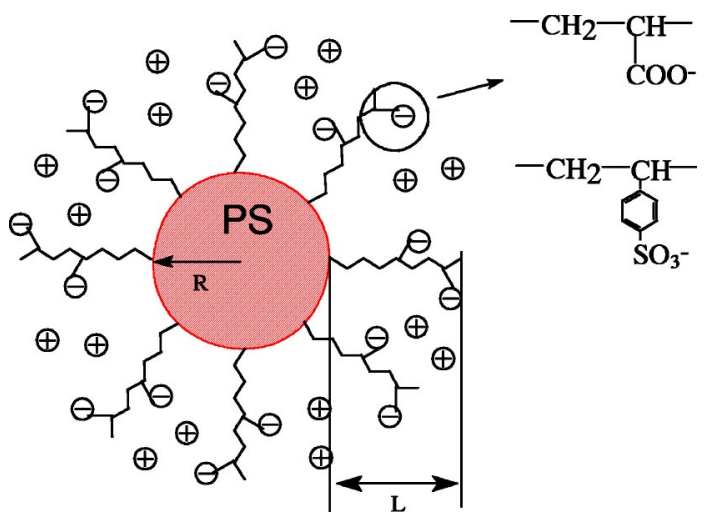

FIG. 1. Schematic representation of the spherical polyelectrolyte brushes investigated herein. Two different types of brushes are investigated here: Quenched brushes where poly(styrenesulfonate) chains (PSS) are grafted to the surface (system Q-SPB), and annealed brushes where poly(acrylic acid) chains are affixed to the surface of the poly(styrene) core particles (system A-SPB). The brush thickness $L$ is a function of the ionic strength within the solution $[17,19]$. 
Bovine Serum Albumine (BSA) adsorb onto these particles in aqueous solution if the ionic strength is low. Little or no adsorption takes place, however, if the ionic strength is high $[10,12]$. If the ionic strength is raised, most of the protein is released. Additional experiments demonstrated that the tertiary structure of the released proteins is mostly maintained [11]. Finally, Fourier-transform infrared (FT-IR) spectroscopy revealed that the secondary structure of several adsorbed proteins including BSA and Bovine Pancreatic Ribonuclease A (RNase A) is only slightly disturbed [14]. This is in excellent agreement with the recent finding that the enzymatic activity of Glucoamylase and other enzymes adsorbed onto SPB is nearly fully retained [13].

The adsorption of negatively charged proteins onto a brush layer consisting of negatively charged polyelectrolyte chains may appear surprising at first: In principle, there is a Coulomb repulsion between the protein and the SPB in solution, in particular at low ionic strength. Moreover, a dense layer of chains affixed to colloidal particles should induce a marked steric repulsion and repel the proteins (see the discussion of steric repulsion in Ref. [16]). The strong adsorption of proteins onto the SPB must therefore be sought in a driving force that can overcome these effects. The previous discussion $[10,12]$ suggests that the adsorption is caused by the counterion release forces: The patches of positive charge on the surface of the proteins become multivalent counterions of the polyelectrolyte chains. Thus, a concomitant number of co- and counterions is thereby released and the entropy of the entire system is increased. A previous discussion by Fleck and von Grünberg [20] has shown that the counterion release force presents a valid concept.

In the case of spherical polyelectrolyte brushes, the counterion release forces are expected to play a major role for the following reasons: Theoretical work predicts that nearly all counterions are confined within the brush layer [15]. The same prediction has been made for the similar case of charged star polymers [21,22]. Hence, the brush layer is practically neutral and its height is dominated by a single force, namely the osmotic pressure of the counterions confined in the layer. If the ionic strength in the solution is low, the polyelectrolyte chains affixed to the surface will be strongly stretched to increase the volume accessible for the counterions and to alleviate their osmotic pressure ("osmotic brush" [15]). On the other hand, added salt screens the electrostatic interaction within the brush layer and the chains behave more or less as neutral polymers ("salted brush" [15]). Experimental work done on planar polyelectrolyte brushes $[23,24]$ and on spherical polyelectrolyte brushes $[17,25]$ has fully corroborated these predictions. Thus, if a protein acts as a multivalent counterion within the brush, the release of monovalent counterions will lower the strong osmotic pressure but retain electroneutrality. Therefore, the adsorption of proteins onto SPB cannot be related to forces usually invoked when explaining the experimental findings related to solid surfaces such as, e.g., hydrophobic effects or van der Waals attraction [3,26].

If the counterion release forces are responsible for the strong attractive interaction of the proteins with the SPB, the proteins cannot be located only at the outer surface but must be dispersed within the brush layer. Here we present a com-
TABLE I. Characterization of the proteins used in this study.

\begin{tabular}{lccccc}
\hline \hline Protein & $\mathrm{Mg} / \mathrm{Mol}^{\mathrm{a}}$ & $\mathrm{pI}^{\mathrm{b}}$ & $R_{g}(\mathrm{~nm})^{\mathrm{c}}$ & $\bar{v}\left(\mathrm{~cm}^{3} / \mathrm{g}\right)^{\mathrm{d}}$ & $\Delta \rho^{\mathrm{e}}$ \\
\hline BSA & 66900 & 5.1 & 3.0 & 0.73 & 304.0 \\
RNase A & 13910 & 9.6 & 1.3 & 0.73 & 302.0 \\
\hline \hline
\end{tabular}

${ }^{\mathrm{a}}$ Molecular weight as determined by SAXS in solution.

${ }^{\mathrm{b}}$ Isoelectric point.

${ }^{\mathrm{c}}$ Radius of gyration as determined by SAXS.

${ }^{\mathrm{d}}$ Partial molar volume as determined from density measurements in dilute solution.

${ }^{\mathrm{e}}$ Contrast of the proteins in water given by number of excess electrons per $\mathrm{nm}^{-3}$ in water.

prehensive investigation of this problem by small-angle x-ray scattering (SAXS $[27,28]$ ). SAXS is well-suited for the study of the SPB under consideration here because of the good contrast between the poly(styrene) core and the brush layer $[29,30]$. Moreover, the monodisperse core particles together with the marked discontinuity of the excess electron density between the core and the shell lead to strong oscillations of the measured scattering intensity $I(q)$ $[q=(4 \pi / \lambda) \sin (\theta / 2)$ is the magnitude of the scattering vector; $\lambda$ is the wavelength of radiation; $\theta$ is the scattering angle]. Proteins in general exhibit a good SAXS contrast when dispersed in water [27] and the adsorption onto the SPB should be easily detected by this method in situ.

Two different proteins, Bovine Serum Albumine (BSA) and Bovine Pancreatic Ribonuclease A (RNase A), have been used in this investigation. The choice of these proteins and the respective carrier particles originates from previous studies: The adsorption of BSA onto annealed SPB has already been the subject of several studies [10-12,14]. The adsorption of RNase A onto a quenched SPB was recently investigated by FT-IR spectroscopy in order to detect possible changes of the secondary structure in the adsorbed state [14]. The analysis of the process of adsorption by SAXS continues these studies inasmuch as it can reveal the location of the adsorbed protein within the brush layer.

\section{EXPERIMENT}

\section{A. Materials}

Bovine Serum Albumine (BSA) and Bovine Pancreatic Ribonuclease A (RNase A) were purchased from Sigma and used without further purification. Table I gathers the main parameters characterizing these proteins.

The synthesis of the spherical polyelectrolyte brushes (SPB) has been done as described recently $[18,19]$. Two SPB have been used in this study: the annealed SPB termed A-SPB carrying chains of poly(acrylic acid) (PAA) and the quenched SPB termed Q-SPB carrying chains of poly(styrene sulfonic acid). The core particles consist of poly(styrene). Table II summarizes the main parameters of these systems. All systems have been purified by exhaustive ultrafiltration. For experiments using BSA, the $p \mathrm{H}$ of the solution was adjusted to 6.1 by $10 \mathrm{mM}$ $\mathrm{N}$-morpholinoethanesulfonic acid (MES) according to the 
TABLE II. Characterization of the spherical polyelectrolyte brushes used in this study.

\begin{tabular}{lcc}
\hline \hline Particle & $R(\mathrm{~nm})^{\mathrm{a}}$ & $L(\mathrm{~nm})^{\mathrm{b}}$ \\
\hline A-SPB & 50 & 54 \\
Q-SPB & 56 & 50 \\
\hline
\end{tabular}

${ }^{\mathrm{a}}$ Core radius of particles.

${ }^{\mathrm{b}}$ Thickness of brush layer (see Fig. 1) determined by DLS at the ionic strength of the adsorption experiments.

method described recently [10]. In the case of RNase A, the $p \mathrm{H}$ was adjusted to 9.3 by $10 \mathrm{mM} \mathrm{2-(cyclohexylamino)-}$ ethanesulfonic acid (CHES). $2 \mathrm{mM} \mathrm{NaN}_{3}$ were added to the buffer solutions in order to avoid microbial growth. The thickness $L$ of the brush layer (see Fig. 1) was determined by dynamic light scattering (DLS) using a Peters ALV 4000 light scattering goniometer [17]. By this method, the overall hydrodynamic radius $R_{H}$ of the particles in the respective solution is determined. The thickness $L$ is then $L=R_{H}-R$, where $R$ denotes the radius of the core particles (see Fig. 1). This subtraction works with high precision since the core particles are practically monodisperse [17]. Table II gathers the data of both types of particles. $L$ was measured directly for the solutions used in the adsorption experiments because this parameter depends sensitively on the ionic strength of the solution [17].

Given amounts of protein were dissolved in aqueous 10 $\mathrm{mM}$ buffer solution containing $2 \mathrm{mM} \mathrm{NaN}_{3}$, and added to the solution of the respective SPB adjusted to the same $p \mathrm{H}$ and stirred for $24 \mathrm{~h}[10,14]$. The fraction of the protein not adsorbed to the SPB was removed by ultrafiltration as described recently [10]. The amount of removed protein was determined by extinction measurements as described elsewhere $[10,14]$.

\section{B. SAXS measurements}

All SAXS data have been measured using a home-built Kratky camera. The details of the measurements and of the desmearing of the data can be found in previous papers $[28,29]$. All scattering curves discussed here present absolute intensities.

\section{RESULTS AND DISCUSSION}

A. Characterization of the proteins and the spherical polyelectrolyte brushes by dynamic light scattering and SAXS

We analyze the scattering function of the spherical polyelectrolyte brushes loaded with different amounts of the respective proteins. It is therefore necessary to analyze first the scattering functions of the proteins and of the SPB under the condition of the measurements. Moreover, the analysis of the radial structure of the composite particles requires special consideration. It is interesting to note that the evaluation of the strongly curved brushes under consideration here resembles closely the analysis of flat brushes by surface reflectivity measurements [23,31-35].

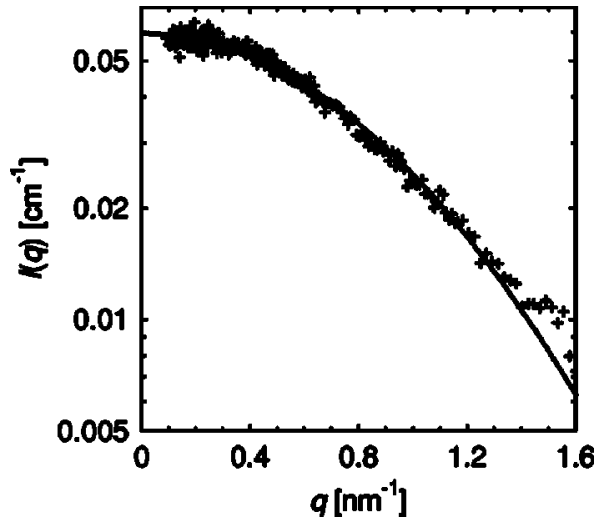

FIG. 2. Analysis of the SAXS intensity of free RNase A in aqueous solution. The weight fraction of RNase A is 0.0056 . The crosses display the measured scattering intensity whereas the solid line gives the optimal fit by Guinier's law [Eq. (1)]. The plot shows that Guinier's law gives a good description of the measured intensities up to $q=1.4 \mathrm{~nm}^{-1}$. The intercept of the solid line yields the molecular weight of the dissolved protein. The value derived from this figure $(13.910 \mathrm{~g} / \mathrm{Mol})$ is in excellent agreement with the theoretical value $(13.700 \mathrm{~g} / \mathrm{Mol})$.

\section{Analysis of the dissolved proteins}

The proteins BSA and RNase A present small objects having a diameter of a few nanometers only. Figure 2 displays the scattering intensity $I(q)$ obtained for a dilute solution of RNase A in the buffer solution. The solid line presents the fit by Guinier's law [27]

$$
I(q) \approx \Delta \rho^{2} c M \exp \left(-\frac{R_{g}^{2} q^{2}}{3}\right),
$$

where $\Delta \rho$ denotes the contrast of the proteins in the solvent water (cf. Table I), $c$ is the weight concentration, $M$ its molecular weight, and $R_{g}$ its radius of gyration. Good agreement is seen up to $q=1.4 \mathrm{~nm}^{-1}$, as expected. The molecular weight obtained from the intercept $(13910 \mathrm{~g} / \mathrm{Mol}$, see Table I) agrees very well with the calculated value (13 700, see Ref. [36]). A similar analysis has been done for BSA in dilute solution (not shown). The agreement of theory (66 472 g/mol, see Ref. [36]) and experiment (66 900, cf. Table I) was similarly satisfying. The main point for the subsequent analysis is the good description of the scattering function of the proteins in terms of Eq. (1) for a $q$ range $q$ $<1.0 \mathrm{~nm}^{-1}$.

\section{Analysis of the spherical polyelectrolyte brushes in solution}

The analysis of SPB in solution has been discussed in great detail recently $[17,19,29,30]$. Dynamic light scattering has revealed that the thickness $L$ of the brush layer (see Fig. 1) depends strongly on the ionic strength in solution $[17,19]$. Two types of SPB have been used here: The annealed SPB A-SPB bearing PAA chains and the quenched SPB Q-SPB carrying PSS chains on the surface of the core particles. In the case of an annealed brush [15,23], the charge density depends strongly on the $p \mathrm{H}$ in the system whereas no dependence is seen for the quenched SPB [17]. The respective $p \mathrm{H}$ 
used in the present experiments, however, is high enough to ensure full titration of all charges in the annealed system [10]. The data of $L$ for the respective SPBs obtained by dynamic light scattering are summarized in Table II. The analysis of the SPB by SAXS was developed in Ref. [29]. The main points are briefly summarized as follows: The intensity $I(q)$ measured at small concentrations can be evaluated disregarding the structure factor $S(q)$ that takes into account the influence of interparticle interferences. This has been argued for similar systems on the basis of model calculations that showed that $S(q)$ alters the scattering curves only at the smallest $q$ values not considered here [28]. Hence, in the following, $S(q)=1$ in excellent approximation.

The scattering intensity $I(q)$ of a single SPB may be split into three terms [29],

$$
I(q)=I_{\mathrm{CS}}(q)+I_{\text {fluct }}(q)+I_{\mathrm{PS}} .
$$

The first term $I_{\mathrm{CS}}(q)$ is due to the overall structure of the SPB that consist of a core with low contrast and a shell having a high SAXS contrast. The shell composed of the polyelectrolyte chains and their counterions is described by an average radial profile directly related to the excess electron density $\rho(r)-\rho_{M}$, where $\rho(r)$ is the radial electron density of the spherical polyelectrolyte brush and $\rho_{m}$ is the electron density of the surrounding medium water. From this it follows that $I_{\mathrm{CS}}(q)=B^{2}(q)$ with the scattering amplitude $B(q)$ given by $[28,29]$

$$
B(q)=4 \pi \int_{0}^{\infty}\left[\rho(r)-\rho_{m}\right] \frac{\sin q r}{q r} r^{2} d r .
$$

Equation (3) can be easily generalized to include polydisperse systems [28]. In this case, the $B_{i}^{2}(q)$ of species $i$ are added up weighed by their respective number density.

The term $I_{\text {fluct }}(q)$ takes into account the fluctuations of the polyelectrolyte layer on the surface [29]. This term has been discussed for a number of systems composed of polymeric layers or networks that are attached to colloidal particles [29,37-39]. In particular, Pedersen and co-workers gave a detailed discussion of the possible modeling of the contribution of attached chains to the scattering function which becomes the leading term at high scattering angles [40-42]. In the present work, however, we are mainly concerning with the region of smaller $q$ values, i.e., with the modeling of $I_{\mathrm{CS}}(q)$. Therefore, a simplified treatment of $I_{\text {fluct }}(q)$ developed in previous investigations $[29,37,39]$ will suffice in the following.

According to Ref. [29], $I_{\text {fluct }}(q)$ may be described by a Lorentzian

$$
I_{\text {fluct }}(q)=\frac{I_{\text {fluct }}(0)}{1+\xi^{2} q^{2}},
$$

where $\xi$ characterizes the spatial extent of the fluctuations. Here it suffices to consider this term as an additional contribution that comes into play mainly at high scattering vectors $q$. The following analysis will show that most of the information can be obtained in a $q$ range in which $I_{\text {fluct }}(q)$ presents a rather small part of the measured intensity.

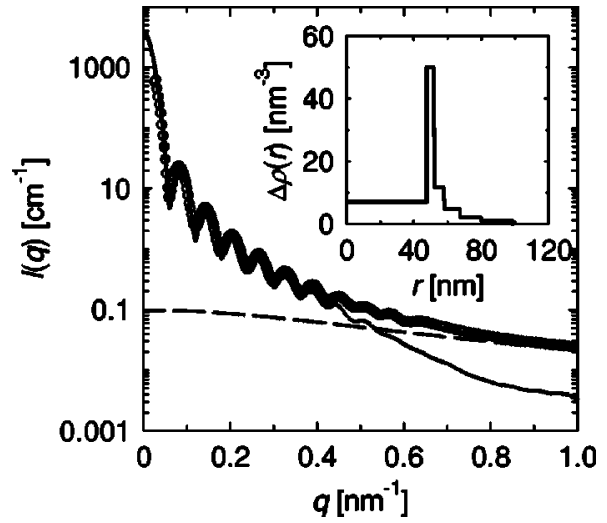

FIG. 3. SAXS intensity of the quenched polyelectrolyte brush Q-SPB (weight fraction: 0.045). The circles give the measured intensity and the solid line shows the optimal fit (see text). The dashed line refers to the contribution to $I(q)$ deriving from the fluctuations of the brush layer affixed to the surface of the particles (see Fig. 1). The inset presents the excess electron density $\rho(r)-\rho_{M}$ as the function of the radial distance $r$ which has been obtained from this fit.

The final $I_{\mathrm{PS}}$ term in Eq. (2) refers to the density fluctuations in the solid core particles (see the discussion in [37]). It is practically negligible for the present $q$ range.

Figure 3 displays the SAXS intensity measured from a dispersion containing 4.5 wt. $\%$ of the quenched SPB Q-SPB. The solution has been adjusted to a $p \mathrm{H}$ of 9.3 and an ionic strength of $10 \mathrm{mM}$ (cf. Sec. II). In this way, the SPB is adjusted for the adsorption experiments using RNase A to be discussed in the next paragraph. It should be noted, however, that the $p \mathrm{H}$ is of no influence for the quenched brush [17]. Therefore, the data shown here can directly be compared to the results presented on a fully titrated annealed brush [29]. The pronounced minima and maxima of the scattering curve displayed in Fig. 3 point directly to the narrow size distribution of the core particles. At higher $q$ values, the scattering curve does not decay as $q^{-4}$, as expected [27]. In this region, the second term of Eq. (2), namely $I_{\text {fluct }}(q)$, becomes the leading term. This is in full accord with previous findings obtained from a related system [29].

In view of the restricted resolution of the SAXS experiment and of the subsequent analysis of the more complicated composite systems, the analysis of $I(q)$ is done here in a slightly different fashion than is described in Ref. [29]. The core radius $R$ is exactly known from a precise analysis of the bare core particles by SAXS. The shell, i.e., the excess electron density $\rho(r)-\rho_{M}$ for radial distance $r>R$, is modeled in terms of five subsequent concentric shell of homogeneous excess electron density. The integral equation (3) hence becomes a sum over five terms for $\rho(r)-\rho_{M}$, whereas the core is modeled by a homogeneous sphere with the excess electron density of poly(styrene) $\left(7 e^{-} / \mathrm{nm}^{3}\right.$, see Ref. [37]). The description becomes much simpler in this way without sacrificing the accuracy of the modeling. In particular, it is welladapted for the analysis of the composite particles (see below). The coarse-grained description leads to artifacts in the scattering curve only at much higher $q$ that are not considered here. Given this simplification of the analysis, the fit of 


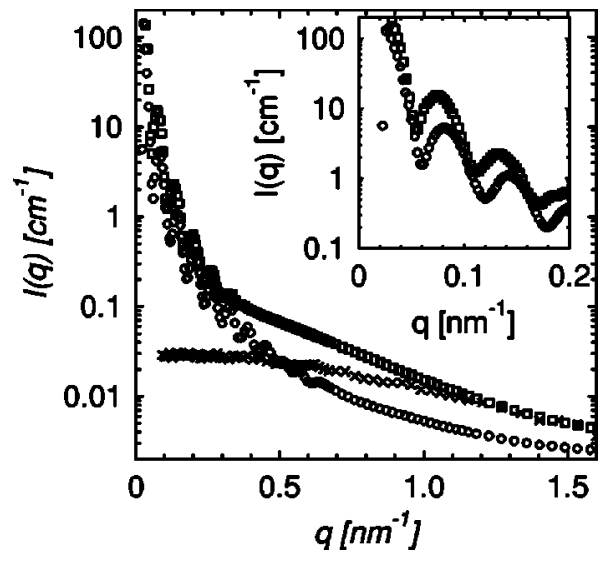

FIG. 4. Comparison of the SAXS intensities of the spherical polyelectrolyte brush Q-SPB (circles), of the same system loaded with RNase A (quadrangles; $408 \mathrm{mg}$ RNase per $\mathrm{g}$ of the carrier particle), and of the free RNase A (crosses; see Fig. 2). Both the scattering curve of the loaded and of the unloaded brush particles has been normalized to the weight fraction. The intensity of the free RNase A has been scaled to the total concentration of the protein adsorbed on the brush particles. The inset shows an enlarged view of the region of smallest angles. Here the shift of the maxima to smaller $q$ values upon absorption of RNase A is clearly seen.

$I(q)$ proceeds along the lines given in Ref. [29] by fitting $I_{\mathrm{CS}}(q)$ and $I_{\text {fluct }}(q)$ in one step. The $q$ ranges in which either term dominates are well-separated (see Fig. 3) and the analysis provides no difficulty. The solid line in Fig. 3 presents a fit of $I_{\mathrm{CS}}(q)$ obtained in this manner, whereas the dashed line displays $I_{\text {fluct }}(q)$ obtained from the fit. The inset displays the excess electron density $\rho(r)-\rho_{M}$ as a function of the radial distance $r$. A full description of the experimental $I(q)$ up to $q=2 \mathrm{~nm}^{-1}$ is achieved in agreement with a previous analysis [29]. For the sake of clarity, only the range up to $q$ $=1 \mathrm{~nm}^{-1}$ is shown. The correlation length was determined to $\xi=1.9 \mathrm{~nm}$ from this fit. A similar magnitude was found previously [29] and presents a reasonable value.

\section{B. Adsorption of proteins on spherical polyelectrolyte brushes}

\section{Modeling of data}

Figure 4 displays a comparison of the scattering intensities of the SPB Q-SPB without RNase A (circles) and after the adsorption of $408 \mathrm{mg}$ RNase A per $\mathrm{g}$ of the SPB (quadrangles). Both intensities have been normalized to the weight fraction of the carrier particles. In addition to this, crosses mark the scattering intensity of free RNase A (see Fig. 2) with a solution containing $0.35 \mathrm{wt} \%$. This concentration refers to the total amount of RNase A in the solution. The shift of the maxima of $I(q)$ of the loaded particles (see the inset of Fig. 4) points directly to an overall increase of the size and the excess electron density within the shell. Moreover, there is an increase of the intensity seen at higher scattering angles. For comparison, the intensity of a solution of free RNase is shown as well. Here it can be seen that the scattering intensities of the loaded particles and of the protein merge at the highest scattering angles. The same behavior is seen for particles loaded with BSA (see Fig. 7).
As is obvious from the contrast factors $\Delta \rho$ gathered in Table I, the proteins exhibit a good SAXS contrast in water and hence contribute markedly to the measured intensity of the composite particles shown in Fig. 4. This leads to the observed increase of the intensity seen at low $q$. At high $q$, the scattering intensity seems to be even dominated by the contribution of the RNase A molecules. A similar behavior has been observed in a SAXS study of a system containing latex particles and micelles $[44,45]$. A modeling of the composite system under consideration here can be done in the same fashion.

At low scattering angles, there is no resolution with regard to the fine details of the shell which are of the order of the size of the proteins. The bound proteins will therefore increase the electron density of the shell but will not be visible as separate entities. In this range, the proteins can be treated similar to adsorbed surfactants [43].

For higher scattering angles, however, this argument is no longer valid. Here the resolution of the SAXS experiment is high enough to distinguish between a continuous electron density and adsorbed spheres. Hence, this region must be treated differently. It can be shown that here the scattering intensities of the SPB and the bound proteins simply add up: In principle, the scattering function of a binary system of spheres of different size originates from the squared sum of the two scattering amplitudes $B(q)$ of the different spheres. Consider the system consisting of one big sphere and several small spheres attached to the surface of the big sphere. The radial averaged scattering intensity of this system follows directly as [27]

$$
\begin{aligned}
I(q)= & B_{\text {large }}^{2}(q)+\sum_{i} B_{\text {small }, i}^{2}(q)+\sum_{i} B_{\text {small }, i}(q) B_{\text {large }}(q) \frac{\sin q d}{q d} \\
& +2 \sum_{i \neq j} B_{\text {small }, i} B_{\text {small }, j} \frac{\sin q d_{i j}}{q d_{i j}}
\end{aligned}
$$

where $d$ denotes the distance between the centers of gravity of the large sphere and a small sphere. The mutual distances between the small spheres are denoted as $d_{i j}$. Hence, the intensity consists of four terms, namely the two scattering intensities of the large and of the small spheres, respectively, and of two additional terms containing the respective mutual distances of the spheres. The first and the third term are functions of $q d$ and will mainly contribute at concomitantly small angles. The first term will fall off as $q^{-4}$ and not play a significant role at higher scattering angles. At higher $q$, the third term is not expected to contribute significantly since $\sin (q d) / q d$ becomes a rapidly oscillating function of $q$ in this region. Hence, a small polydispersity already will suffice to cancel the contribution of this term at sufficiently high $q$. Because of the low resolution of the SAXS experiment at low $q$, the first sum in Eq. (5) can be described in terms of an increased electron density located on the surface of the large sphere. This will become more obvious when considering the small but finite width of the size distribution of the core particles that leads to a distribution of $d$ as well. The second term is simply the intensity of a suspension of noninteracting 
small spheres. Evidently, this term will be a leading contribution at high $q$.

It remains to discuss the magnitude of the second sum in Eq. (5). We proceed from the assumption that the proteins modeled by the small spheres are distributed at random within the shell. Thus, the distance $d_{i j}$ will be a random variable and the average over the last term in Eq. (5) will vanish. However, a finite correlation between the spheres within the shell must still exist because the minimum distance between two small spheres must be their diameter. This distance $d_{\text {min }}$ must be of the order of $2 R_{g}$ of the proteins. All alterations affected by this "correlation hole" can only appear at higher scattering angles $\left(q>1 \mathrm{~nm}^{-1}\right)$ not under consideration here. These considerations may appear difficult because they are subject to a number of approximations. Since the subsequent evaluation of the data rests fully on these arguments, the Appendix presents a model calculation that gives a proof that this method is valid indeed.

Hence, the subsequent analysis proceeds as follows: In the region of small scattering angles, the adsorbed protein is assumed to enhance the radial electron density within the shell of the particles. Therefore, the calculation of $I_{\mathrm{CS}}(q)$ of the composite particles is done by increasing the excess electron density of the shells defined previously in the analysis of the unloaded particles. Here different models can be used that take into account the correlation of the local density of the adsorbed protein molecules to the radial density of the polyelectrolyte chains: The local density of the protein may, e.g., be assumed to be proportional to the local density of the segments of the polyelectrolyte chains. As an alternative, it may be assumed that most of the protein will be adsorbed in the vicinity of the core particles or only at the periphery. The analysis of $I_{\mathrm{CS}}(q)$ in terms of five subsequent concentric shells is very useful for this analysis because it minimized the number of free parameters of such a fit. For the different models just mentioned, it suffices to define a relation between the segment density of the polyelectrolyte chains in a given shell and the amount of protein located in this particular shell.

The adsorption of large amounts of protein may lead to an extension of the spatial dimensions of the shell of the SPB. In order to account for this effect, the radial width of the stripes describing the radial excess electron density $\Delta \rho(r)$ $-\rho_{m}$ can be slightly increased if necessary. Since SAXS measurements are sensitive to the overall size of the particles, the modeling of the composite particles gives the information about a possible extension of the shell as well. This extension is certainly to be expected in the case of large bulky protein molecules such as, e.g., BSA (see below).

The core-shell part $I_{\mathrm{CS}}(q)$ of the intensity $I(q)$ thus obtained will decay at least as $q^{-4}$ and be very small beyond $q>0.4 \mathrm{~nm}^{-1}$. As a consequence of this, $I(q)$ at higher scattering angle will be modeled as the sum of the Lorentzian Eq. (4) and the scattering intensity of a solution of the free RNase A having the same concentration as the adsorbed protein. The scattering function of the proteins is taken directly from the analysis of the free systems shown for RNase A in Fig. 2. Hence, the contribution of the proteins to the measured $I(q)$ is modeled by Eq. (1). As suggested by the analy-

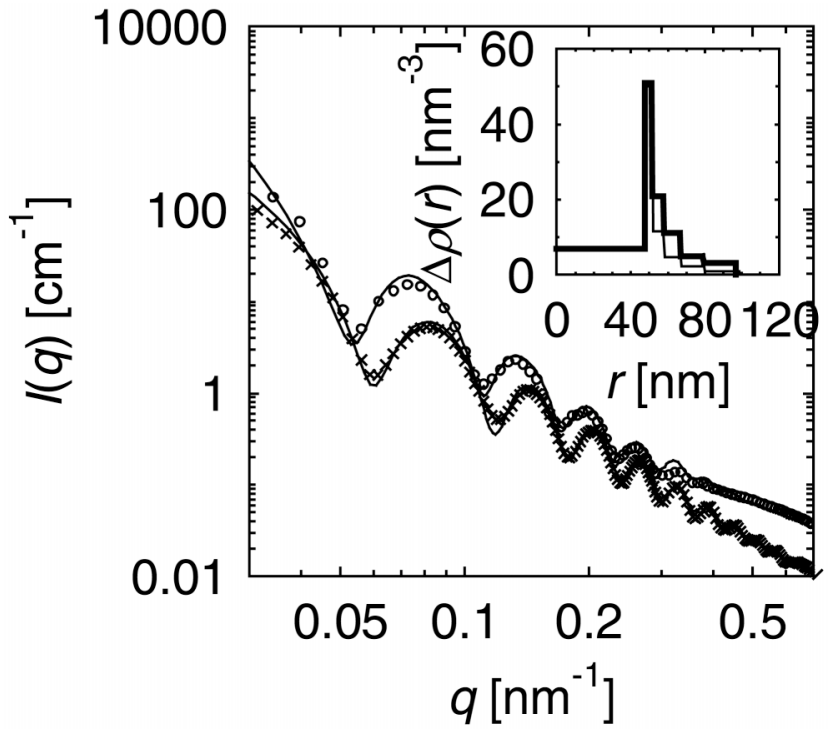

FIG. 5. Optimal fit of the SAXS intensity of the quenched polyelectrolyte brush Q-SPB onto which RNase A has been adsorbed. Crosses display the scattering intensity of the unloaded particles whereas circles give the intensity of the loaded particles. The amount of adsorbed RNase A is $408 \mathrm{mg}$ per $\mathrm{g}$ of the carrier particle. Both curves have been normalized to the weight fraction of the carrier particles. The inset gives the radial distribution of the excess electron density of the brush particle (thin line) and of the brush particle + RNase (thick line). See text for further explanation.

sis shown in Fig. 4, the concentration $c$ of the protein is rescaled to match its total concentration in solution.

For the sake of simplicity, the correlation length of the Lorentzian Eq. (4) has been taken from the analysis of the unloaded SPB. Evidently, there may be a change of $\xi$ when the protein is absorbed. The subsequent analysis will show, however, that the contribution of the adsorbed protein is much larger than the fluctuation-induced part embodied in Eq. (4), at least for the range of scattering angles considered here. Therefore, possible changes will not be visible in the $q$ range under scrutiny in the present analysis. Hence, the overall analysis can be done with a minimum of adjustable parameters inasmuch as $R_{g}$ of the proteins and $\xi$ of the carrier particles are assumed to remain unaltered when the protein is absorbed. The only fit parameters used here refer to the alteration of the core-shell structure as given by $I_{\mathrm{CS}}(q)$.

Evidently, the radial distribution $\Delta \rho(r)-\rho_{m}$ of the loaded particles must be related to the overall amount of adsorbed protein. With the known excess electron densities of the proteins, the zeroth moment of $\Delta \rho(r)-\rho_{m}$ must directly give the total amount of protein within the brush layer. This can be compared to the amount of adsorbed protein determined directly by the method devised previously [10].

\section{RNase A: Results}

Figure 5 displays the optimal fit obtained in this way. The fit is calculated from this profile and a standard deviation of $6.5 \%$ for the diameter of the core. The radial distribution following from this fit is shown in the inset of Fig. 5. At first a trial distribution of the absorbed protein was assumed 


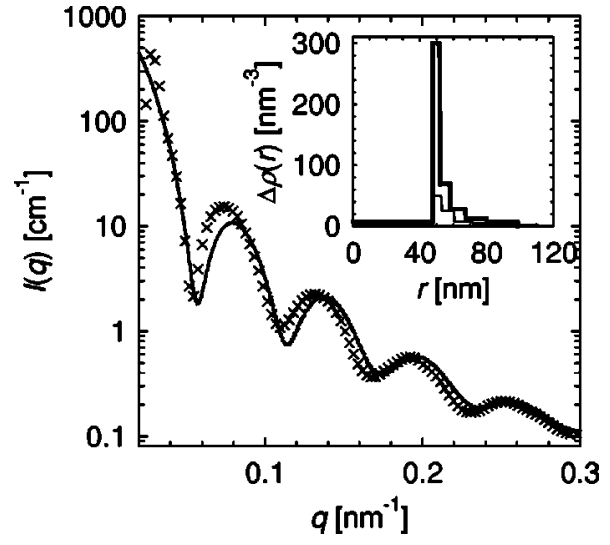

FIG. 6. Locus of adsorption of RNase inside of the brush layer of the quenched spherical polyelectrolyte brush Q-SPB. Circles give the measured scattering intensity of the loaded particles (see Fig. 5). The thin line in the inset gives the radial distribution of the unloaded particle whereas the thick line shows the distribution used for the calculation of the intensity (solid line). In this model, a preferential adsorption near the surface of the core particle was assumed. The obtained fit (solid line) shows poor agreement with the experimental data displayed by circles. Comparing this result to Fig. 5 demonstrates that this model can be ruled out. See text for further explanation.

which follows the distribution of the polyelectrolyte chains. This distribution was changed until the calculated scattering intensity matches the experimental result. The thick line in the inset of Fig. 5 was found to be optimal excess electron density from this fit, whereas the thin line in the inset of Fig. 5 displays the same quantity for the unloaded shell. All parameters taken from the analysis of the carrier particles as well as of the protein have been kept constant. Excellent agreement is found. This distribution describes the experimental data up to $q=0.6 \mathrm{~nm}^{-1}$. Moreover, the amount of adsorbed protein that follows from this fit and subsequent results agrees with the directly determined value within the limits of error $(\sim 10-15 \%)$.

This result demonstrates that the polyelectrolyte chains are solely responsible for the adsorption of the proteins; no particular interaction of the proteins with the surface of the core particles intervenes. The present fit even suggests that RNase A avoids a direct contact with the surface. This can be deduced from the fact that the increase of the excess electron density in the immediate vicinity of the surface (see the inset of Fig. 5) remains nearly unchanged when going from the bare particle to the loaded particle. This interesting finding that may be deduced from the present fits will be discussed further below when presenting the data obtained with BSA.

The validity of this fit can be checked by considering different models assuming a different correlation of the proteins and the polyelectrolyte chains in the shell of the particles. Figure 6 displays the fit in which a much higher density of the protein has been allocated in the first shell above the surface of the core particle (see the inset of Fig. 6). Therefore, the density in subsequent shells must be concomitantly lower since the zeroth moment of the entire shell, i.e., the amount of adsorbed protein, must not change. The fit of the experimental intensity has become much worse and this

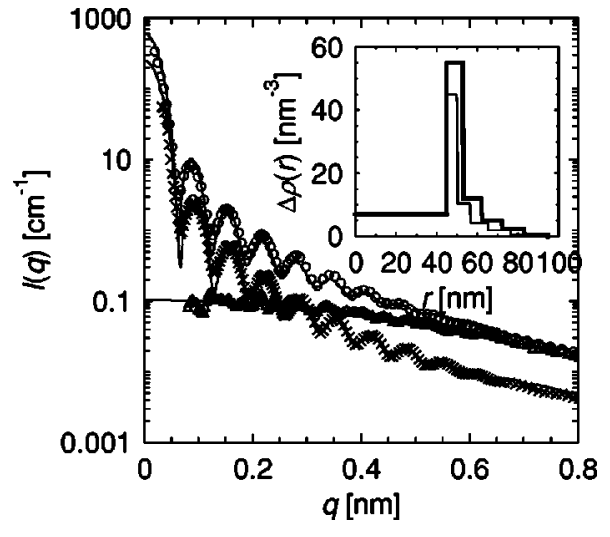

FIG. 7. Adsorption of BSA onto the annealed spherical polyelectrolyte brush A-SPB. The crosses give the scattering intensity of the unloaded particles whereas the circles refer to $296 \mathrm{mg}$ BSA per $\mathrm{g}$ of the carrier particles. Both intensities are normalized to the weight fraction of the carrier particles. The inset gives the respective fits of the radial distribution of the excess electron densities. The triangles mark the intensity of an aqueous solution of free BSA scaled up to match the weight concentration of the adsorbed BSA molecules.

model can be ruled out easily. Additional attempts to fit a model that assumes the adsorption of most of the protein in the periphery of the brush particles (not shown) lead to even much worse fits. All data demonstrate that the radial distribution of RNase A follows the radial density of the PAA chains. This points clearly to the fact that the adsorption is not related to the interaction of the proteins with the surface but only to the interaction of the proteins to the polyelectrolyte chains.

All facts found here show that the SPB present a relatively mild way of immobilizing RNase A. Virtually no direct contact with a hydrophobic surface takes place, but the protein molecules are suspended within the brush layer. It is therefore expected that the secondary structure of RNase A is only slightly disturbed during the process of adsorption if at all. This is found indeed in a recent study by FT-IR [14].

\section{3. $B S A$}

Previous work has shown that BSA has a strong affinity to spherical polyelectrolyte brushes if the ionic strength in the solution is low [10]. More than one gram of BSA could be adsorbed per gram carrier particle under these conditions. Nearly full desorption takes place, however, if the ionic strength is raised. The tertiary structure of the desorbed BSA is mostly preserved [11]. Moreover, a recent study by FT-IR demonstrated that the secondary structure of the adsorbed BSA is hardly disturbed. BSA is therefore well-suited for adsorption studies on SPB.

BSA is a much larger protein and its $R_{g}$ is more than twice the one of RNase A (see Table I). Adsorption of this protein is therefore expected to change the scattering curves of the SPB in an even more marked way than was observed in the case of RNase A (see Fig. 5). The same analysis has been done for particles loaded with BSA. Here the annealed SPB particles A-SPB have been used (cf. Table II). Figure 7 dis- 


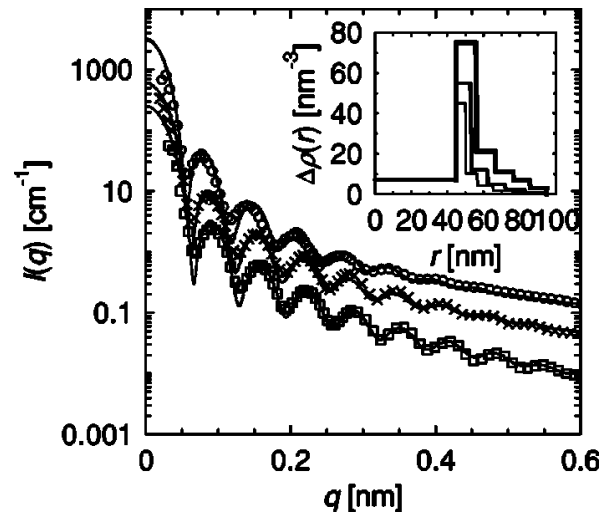

FIG. 8. Adsorption of BSA onto the annealed spherical polyelectrolyte brush A-SPB. The open squares mark the scattering intensity obtained for the unloaded brush. The crosses refer to $296 \mathrm{mg}$ per $\mathrm{g}$ of the carrier particle while the circles refer to $1116 \mathrm{mg}$ per $\mathrm{g}$ carrier particle. All intensities have been normalized to the weight fraction of the carrier particles. The inset gives the respective fits of the radial distribution of the excess electron densities.

plays the measured intensity obtained after adsorbing 296 mg BSA per gram carrier particle. Again it is observed that the rescaled scattering curve of the free protein merges with $I(q)$ of the composite particles at higher scattering angles. At low scattering angles, there is the marked shift of the maxima of the scattering curves to smaller $q$ values because of the increase of electron density within the subsequent shells. The features have already been observed in the case of RNase A and the analysis of the composite particles may proceed in the same way. Because of the larger dimensions of BSA, it turned out to be necessary to increase the thickness of the subsequent shells used for the modeling of the adsorption (see the inset of Fig. 7). Attempts to use the same thickness already defined for the analysis of the unloaded particles failed to produce acceptable fits. This points clearly to an increase of the overall volume of the shell due to the adsorption of the much bulkier BSA molecules.

Figure 8 demonstrates the change of $I(q)$ upon adsorption of more and more BSA. The upper-most curve refers to an adsorption of more than one gram of the protein per gram carrier particle. Here a marked increase of the overall size of the composite particles is expected and seen indeed, as becomes obvious from the inset. The maxima of $I(q)$ are increasing and move to smaller $q$ at the same time in a defined manner. This points to the increase of the electron density in the subsequent shell upon adsorption of BSA.

Different models were compared to these results. As already found in the case of RNase A, the local density of BSA follows closely the radial segment density. Other models assuming a much higher protein density either at the surface or at the periphery failed to fit the positions as well as the height of the maxima of $I(q)$.

Both sets of data obtained from totally different proteins, namely RNase A and BSA, hence demonstrate that the adsorption of proteins is solely due to their interaction with the polyelectrolyte chains. Therefore, the proteins are entering the brush layer and distribute inside the shell according to the segment density of the polyelectrolyte chains with which

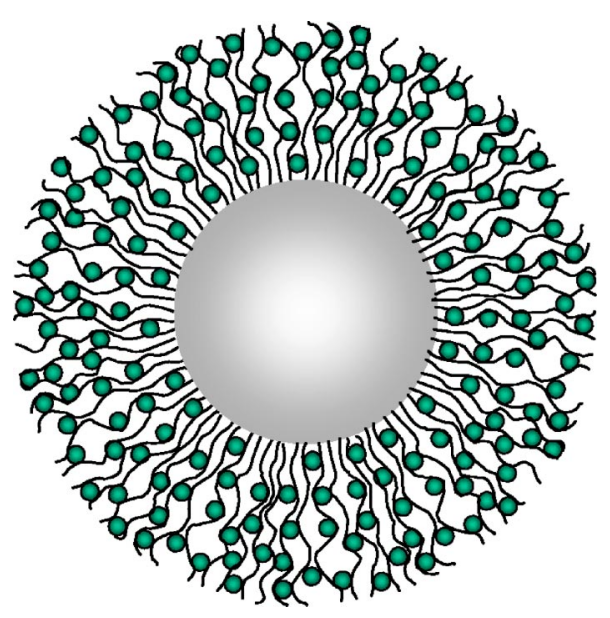

FIG. 9. Schematic representation of the spherical polyelectrolyte brushes loaded with protein. See text for further explanation.

they interact. This is opposite to the findings obtained by Tran et al. [34] for fibrinogen adsorbed to planar polyelectrolyte brushes. Here the protein enters only the periphery of the brush as becomes obvious from an analysis of the segment density by $\mathrm{x}$-ray reflection. The reason for the different behavior is not known. Studies using fibrinogen and the SPB under consideration here must be done to resolve this question.

Both Figs. 7 and 8 point to the fact that BSA may also be adsorbed in the immediate neighborhood of the surface of the SPB. This can be argued from the fact that the excess electron density of the loaded particles is also increased in the first stripe describing the excess electron density directly above the surface of the core particles (see Fig. 8). This finding is opposite to the results obtained with RNase A discussed in the preceding sections. Additional checks showed that the difference between the two proteins is significant and beyond the limits of error. The reason for the different behavior of the two proteins is not yet clear.

\section{CONCLUSION}

An investigation of the adsorption of two proteins, RNase A and BSA, onto spherical polyelectrolyte brushes by SAXS has been presented. The main result of the present analysis is the proof that the proteins enter into the brush layer. Figure 9 displays the findings obtained here for BSA in a schematic fashion. Both the SPB and the protein BSA are drawn to scale. Figure 9 displays the case of BSA inasmuch as the adsorption near the surface of the core particles is finite. All results demonstrate that both proteins enter deeply into the polyelectrolyte brush layer as shown schematically in Fig. 9. In the case of RNase A, however, the protein concentration directly at the surface is smaller as compared to the case of BSA.

Hence, the present study clearly demonstrates that adsorption of the proteins is due to the interaction of the polyelectrolyte chains with the biomolecules. As already discussed by us previously [10], the "counterion release forces" [20] are the driving force for the adsorption of proteins to polyelec- 


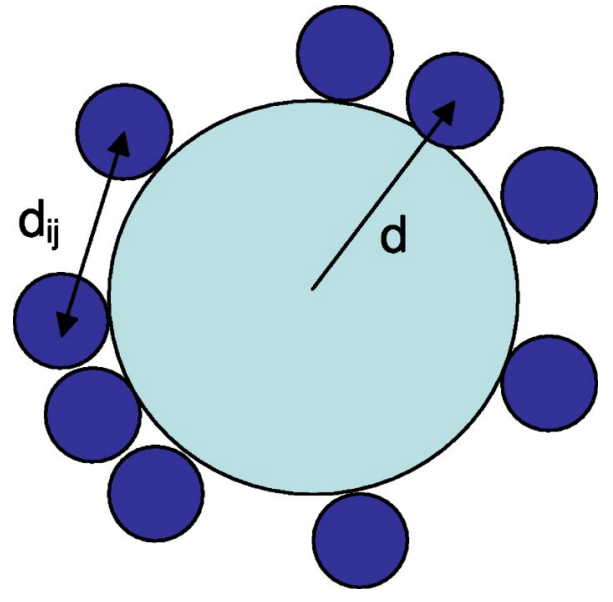

FIG. 10. Scheme of the model used for the calculation of the scattering function of the composite system consisting of the SPB and adsorbed protein. See text for further explanation.

trolyte brushes. The positive patches of the proteins can become multivalent counterions of the charged segments of the poly(acrylic acid) or the poly(styrene sulfonic acid). In this way, the respective counterions of both the patches as well as of the polyelectrolyte segments are liberated, thus releasing the high osmotic pressure within the brush layer. Adsorption of proteins by spherical polyelectrolyte brushes therefore presents a novel way to immobilize proteins.

\section{ACKNOWLEDGMENTS}

We wish to acknowledge the financial support by the Deutsche Forschungsgemeinschaft, the Roche Company, and the BASF AG.

\section{APPENDIX: CALCULATION OF THE SCATTERING FUNCTION}

It the following, model calculations are described which justify the evaluation of data used herein. Figure 10 shows schematically the model: We consider a large sphere of 50 $\mathrm{nm}$ radius (contrast: $7 e^{-} / \mathrm{nm}^{3}$ ) onto which a single layer of monodisperse, much smaller spheres (diameter: $6 \mathrm{~nm}$, contrast: $30 e^{-} / \mathrm{nm}^{3}$ ) is attached at random. The attachment of a given sphere is done by choosing a certain solid angle. If the place on the surface of the large sphere thus chosen is already occupied, a new solid angle is chosen at random. If the place is free, the sphere is attached directly to the surface. Hence, all small spheres have the same distance $D$ to the center of the large spheres but their mutual position is uncorrelated. In this way, various degrees of surface coverage can be achieved. Given the positions of the various spheres, the scattering function $I(q)$ of the entire composite can be calculated exactly by resorting to Eq. (5).

In order to account for the polydispersity of the large spheres, i.e., of the cores of the SPB, the above calculation

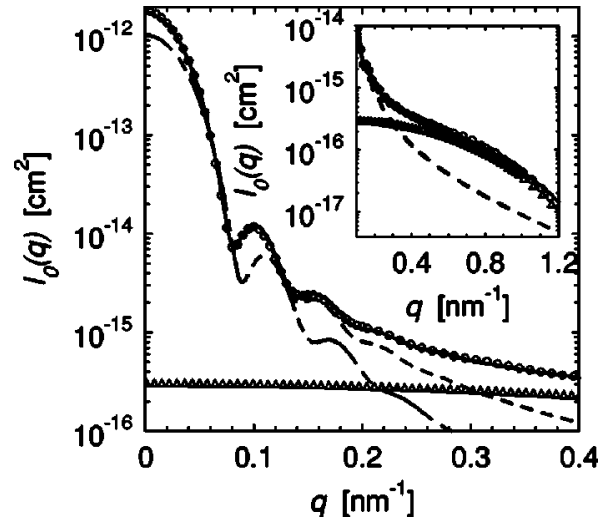

FIG. 11. Calculation of the scattering cross section of the composite system consisting of the SPB and adsorbed protein. The circles mark the exact $I(q)$ obtained by the simulation. The triangles mark $I(q)$ deriving from a ensemble of uncorrelated spheres. The short-dashed line gives the intensity of a core shell system in which the small spheres only constitute a shell of additional electron density around the large sphere. The long-dashed line shows $I(q)$ of the large sphere in the center. The solid line marks the sum of the core-shell calculation (short-dashed line) and the intensity of the free small spheres (triangles). The inset shows the full $q$ range to demonstrate that the intensity of the small spheres is governing the range of higher scattering angles. See text for further explanation.

has been repeated for a set of large spheres differing in size. The scattering function of the composite particles has been added up weighted by their respective number density. For the model calculation shown here, a Gaussian distribution of the large spheres having a polydispersity of $10 \%$ has been assumed. Thus, $I(q)$ shown by circles in Fig. 11 derives from a large number of composite spheres, which ensures good statistics.

Figure 11 displays the resulting scattering cross section (circles). The triangles mark the scattering originating from an ensemble of the same number of small spheres dispersed at random. Evidently, the scattering intensity of the composite system coincides with the intensity of the ensemble of small spheres. Only at much higher $q$ values is there a discrepancy because of the first minimum of $I(q)$ of the small spheres (at $q=1.5 \mathrm{~nm}^{-1}$ ). Since this region of scattering angles is beyond the range of $q$ considered here, this deviation of the exact curve and the model is inconsequential.

At small $q$ values, the core-shell approach marked by the short-dashed line gives a good fit. The long-dashed curve marks $I(q)$ of the big sphere in the core. The shift of the core-shell system is clearly seen. Both intensities must decay with $q^{-4}$ at higher scattering angles. The solid line describes the sum of the core-shell model (short-dashed line) and the intensity of the small spheres (triangles). Evidently, the scattering intensity $I(q)$ of the composite system is well described by the sum of these terms for $q<1.2 \mathrm{~nm}^{-1}$. The scattering intensity of the SPB having adsorbed protein on the surface can hence be described within this approximation. 
[1] Physical Chemistry of Biological Interface, edited by A. Baszkin and W. Norde (Marcel Dekker, New York, 1999).

[2] C. A. Haynes and W. Norde, Colloids Surf., B 2, 517 (2000).

[3] Biopolymers at Interfaces, edited by M. Malmstens (Marcel Dekker, New York, 2003).

[4] E. Ostuni, R. G. Chapman, R. E. Holmlin, S. Takayama, and G. M. Whitesides, Langmuir 17, 5605 (2001), and further references given there.

[5] H. Kawaguchi, Prog. Polym. Sci. 25, 1171 (2000).

[6] T. Zougrana, G. H. Findenegg, and W. Norde, J. Colloid Interface Sci. 190, 437 (1997).

[7] C. Czeslik, C. Royer, T. Hazlett, and W. Mantulin, Biophys. J. 84, 2533 (2003).

[8] S. D. Chakarova and A. E. Carlsson, Phys. Rev. E 69, 021907 (2004).

[9] See, e.g., the recent study of the protease/clay interaction: B. P. Kelleher, S. F. Oppenheimer, F. X. Han, K. O. Willeford, M. J. Simpson, A. J. Simpson, and W. L. Kingery, Langmuir 19, 9411 (2003).

[10] A. Wittemann, B. Haupt, and M. Ballauff, Phys. Chem. Chem. Phys. 5, 1671 (2003).

[11] G. Jackler, A. Wittemann, M. Ballauff, and C. Czeslik, Spectroscopy (Eugene, Or.) 18, 289 (2004).

[12] C. Czeslik, R. Jansen, M. Ballauff, A. Wittemann, C. A. Royer, E. Gratton, and T. Hazlett, Phys. Rev. E 69, 021401 (2004).

[13] Th. Neumann, B. Haupt, and M. Ballauff, Macromol. Biosci. 4, 13 (2004).

[14] A. Wittemann and M. Ballauff, Anal. Chem. 76, 2813 (2004).

[15] P. Pincus, Macromolecules 24, 2912 (1991).

[16] G. J. Fleer, M. A. Cohen Stuart, J. M. H. M. Scheutjens, T. Cosgrove, and B. Vincent, Polymers at Interfaces (Chapman and Hall, London, 1993).

[17] X. Guo and M. Ballauff, Phys. Rev. E 64, 051406 (2001).

[18] X. Guo, A. Weiss, and M. Ballauff, Macromolecules 32, 6043 (1999).

[19] X. Guo and M. Ballauff, Langmuir 16, 8719 (2000).

[20] C. Fleck and H. H. von Grünberg, Phys. Rev. E 63, 061804 (2001); see also A. A. Meier-Koll, C. C. Fleck, and H. H. von Grünberg, J. Phys.: Condens. Matter 16, 6041 (2004).

[21] A. Jusufi, C. N. Likos, and H. Löwen, Phys. Rev. Lett. 88, 018301 (2002).

[22] A. Jusufi, C. N. Likos, and H. Löwen, J. Chem. Phys. 116, 11011 (2002).
[23] H. Ahrens, S. Förster, and C. A. Helm, Phys. Rev. Lett. 81, 4172 (1998).

[24] M. Biesalski, D. Johannsmann, and J. Rühe, J. Chem. Phys. 117, 4988 (2002).

[25] B. Das, X. Guo, and M. Ballauff, Prog. Colloid Interf. Sci. 121, 34 (2002).

[26] A. R. Mackie, J. Mingins, and A. N. North, J. Chem. Soc., Faraday Trans. 87, 3043 (1991).

[27] Small-Angle X-ray Scattering, edited by O. Glatter and O. Kratky (Academic Press, London, 1982).

[28] N. Dingenouts, J. Bolze, D. Pötschke, and M. Ballauff, Adv. Polym. Sci. 144, 1 (1998).

[29] Q. de Robillard, X. Guo, M. Ballauff, and T. Narayanan, Macromolecules 33, 9109 (2000).

[30] N. Dingenouts, R. Merkle, X. Guo, T. Narayanan, G. Goerigk, and M. Ballauff, J. Appl. Crystallogr. 36, 578 (2003).

[31] P. Auroy and L. Auvray, J. Phys. II 3, 227 (1993).

[32] P. Auroy and L. Auvray, Langmuir 10, 225 (1994).

[33] P. Auroy and L. Auvray, Macromolecules 29, 337 (1996).

[34] Y. Tran, P. Auroy, L.-T. Lee, and M. Stamm, Phys. Rev. E 60, 6984 (1999).

[35] F. Dubreuil and P. Guenoun, Eur. Phys. J. E 5, 59 (2001).

[36] The sequences of BSA and of RNase A have been taken from the Protein Data Bank: H. M. Berman, J. Westbrook, Z. Feng, G. Gilliland, T. N. Bhat, H. Weissig, I. N. Shindyalov, and P. E. Bourne, The Protein Data Bank. Nucleic Acids Research 28, 235 (2000). Identification codes: RNase A: 1AQP; BSA: 1 AO6.

[37] N. Dingenouts, Ch. Norhausen, and M. Ballauff, Macromolecules 31, 8912 (1998).

[38] N. Hatto, T. Cosgrove, and M. J. Snowden, Polymer 41, 7133 (2000).

[39] S. Seelenmeyer, I. Deike, S. Rosenfeldt, Ch. Norhausen, N. Dingenouts, M. Ballauff, T. Narayanan, and P. Lindner, J. Chem. Phys. 114, 10471 (2001).

[40] J. S. Pedersen and M. C. Gerstenberg, Macromolecules 29, 1363 (1996).

[41] J. S. Pedersen, J. Chem. Phys. 114, 2839 (2001).

[42] M. Stieger, W. Richtering, J. S. Pedersen, and P. Lindner, J. Chem. Phys. 120, 6197 (2004).

[43] S. Seelenmeyer and M. Ballauff, Langmuir 16, 4094 (2000).

[44] J. Bolze, K. D. Hörner, and M. Ballauff, Langmuir 12, 2906 (1996).

[45] J. Bolze, K. D. Hörner, and M. Ballauff, Colloid Polym. Sci. 274, 1099 (1996). 